\section{Dihydrodipicolinate Synthase Deficiency of Brevibacterium flavum Strain BB69, a Threonine-producing Mutant with a Feedback-resistant Homoserine Dehydrogenase}

\author{
Atsushi YoKota* and Isamu SHIIO** \\ Central Research Laboratories, Ajinomoto Co., Inc., \\ Kawasaki-ku, Kawasaki, Kanagawa 210, Japan
}

Received August 28, 1989

In the genus Brevibacterium, the following two types of threonine producers have been reported: mutants with $\operatorname{HD}^{\mathrm{R}} \mathrm{s}^{1,2)}$ and those with a DPS ${ }^{-}$or DPS $\mathrm{S}^{\mathrm{L}, 4)}$ (Fig. 1). All of them were derived as threonine-producing mutants resistant to AHV, a threonine analogue. The former are the conventional producers, while the latter are the novel producers which have been found by our recent studies ${ }^{3,4}$ for the first time in the coryneform bacteria. So far, it has been shown that the threonine yields of simple $\mathrm{HD}^{\mathrm{R}}$ or $\mathrm{DPS}^{-i \mathrm{~L}}$ type producers are almost the same, i.e., not better than around $10 \mathrm{~g} / 1$ in the various kinds of production media containing $10 \%$ glucose ${ }^{1,3)}$ However, the threonine productivity of a typical $\mathrm{HD}^{\mathrm{R}}$ type threonine producer, $B$. flavum strain BB69, which was derived from a wild strain No. 2247 (ATCC14067) by two-step mutagenesis as a mutant with increased AHV resistance, was elevated to $13.5 \mathrm{~g} / \mathrm{I}^{13}$ Thus in this study, the strain BB69 was examined as to the mechanism of threonine production including its DPS activity.

Table I shows the activities of DPS and HD, and the threonine productivity of strain BB69. The DPS activity of strain BB69 was found to be reduced to about one-tenth that of the original strain No. 2247, and its HD was confirmed to be feedback-resistant. Thus, strain BB69 was found not to be a typical $H^{R}$ type producer, but had both an $\mathrm{HD}^{\mathrm{R}}$ and $\mathrm{a} \mathrm{DPS}^{\mathrm{L}}$. In general, the growth and threonine

Table I. Properties of $\alpha$-Amino- $\beta$-hydroxyvaleric ACID-Resistant Threonine-Producing Strains, BB69 and DK 131, Derived from the Wild Strain No. 2247 and

Aspartate-PRoducing Strain No. 70, Respectively

The threonine production of strain BB69 was examined by the same method as that used for strain DK 131 , i.e., method $\mathrm{B}^{3)}$ using Medium T2 (N30 M4) as the basal medium. The DPS activity was measured as described previously. ${ }^{3)}$ The HD activities of strains No. 2247 and BB69 were measured as described previously ${ }^{5)}$ with cells. cultured by the same method as that used for the threonine production for strain $B B 69^{3)}$ except that the cultivation was done in Medium T2 (N20 M4) for $40 \mathrm{hr}$.

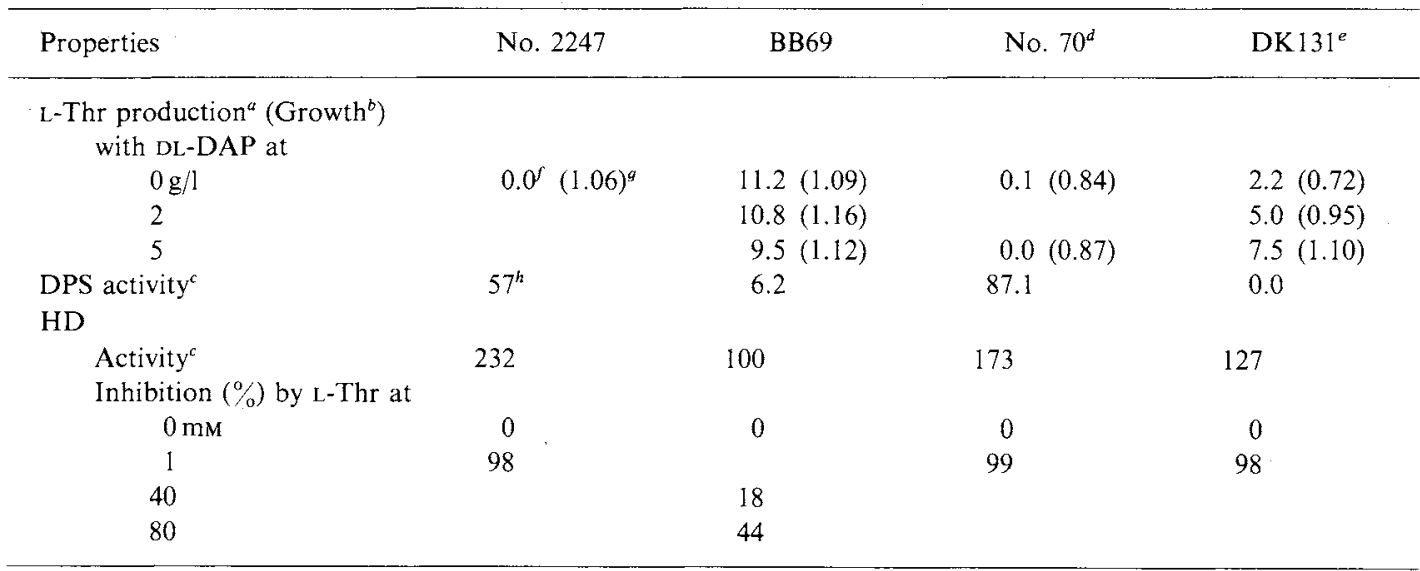

a $/ \mathrm{l}$.

b Absorbance at $562 \mathrm{~nm}$ of the culture broth after 26 -fold dilution with $0.1 \mathrm{~N} \mathrm{HCl}$ solution.

c $\mathrm{nmol} / \mathrm{min} / \mathrm{mg}$ protein.

d.e,f,g,h Data from refs. $4,3,1,6$, and 7 , respectively.

Abbreviations: HD, homoserine dehydrogenase; $\mathrm{HD}^{\mathrm{R}}$, feedback-resistant $\mathrm{HD} ; \mathrm{DPS}$, dihydrodipicolinate synthase; DPS $^{-}$, DPS defect; DPS ${ }^{\mathrm{L}}$, low level DPS; DAP, diaminopimelic acid; AHV, $\alpha$-amino- $\beta$-hydroxyvaleric acid

* Present address: Department of Agricultural Chemistry, Faculty of Agriculture, Hokkaido University, Sapporo 060. Japan.

** Corresponding author. 


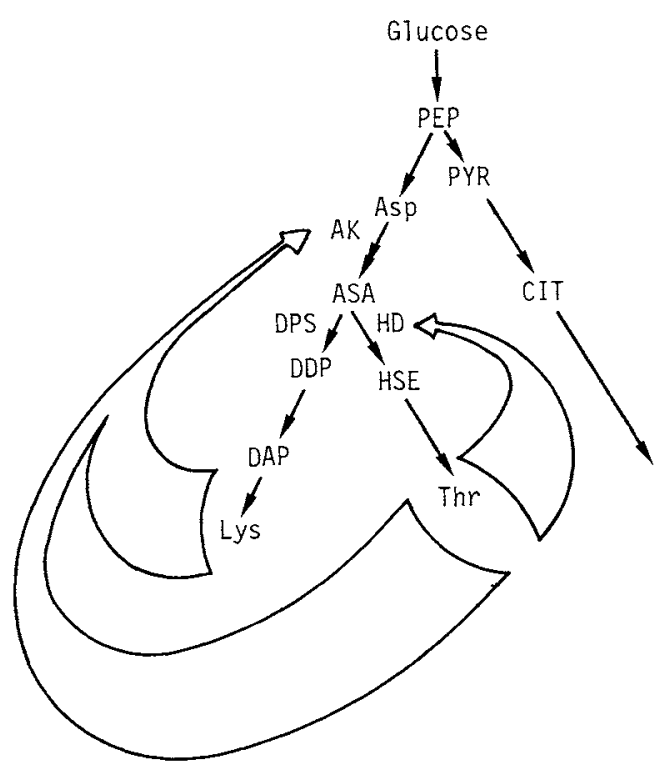

Fig. 1. Threonine and Lysine Biosyntheses and Their Regulation in Brevibacterium flavum.

PEP, phosphoenolpyruvate; ASA, aspartate- $\beta$-semialdehyde; DDP, dihydrodipicolinate; HSE, homoserine; PYR, pyruvate; CIT, citrate; $\mathrm{AK}$, aspartokinase; $\Rightarrow$, feedback inhibition.

productivity of DPS ${ }^{-}$type threonine producers are enhanced by DAP, which is represented by a simple DPS ${ }^{-}$ mutant, strain DK131, ${ }^{3 !}$ derived from strain No. 70 , as shown in Table I. However, those of strain BB69 were not enhanced by DAP, and the productivity was rather decreased by the addition of $5 \mathrm{~g} / \mathrm{l}$ of DL-DAP. They were considered to be attributed to its DPS ${ }^{L}$ character, i.e., strain
BB69 was not deficient in DAP, thus in the presence of the excess DAP, threonine and lysine that was formed from the DAP concertedly brought about the feedback inhibition of the aspartokinase. As reported in our preceding paper ${ }^{4}{ }^{4}$ even among the simple DPS ${ }^{-}$strains derived from strain No. 70 , the growth enhancement by DAP was not remarkable in strains DK203 and DK330 compared with strain DK131. Thus, it is not irrational that the growth of strain BB69 with a DPS ${ }^{\mathrm{L}}$ was not enhanced by DAP.

The above results suggest the usefulness of DPS mutations in combination with the $H D^{R}$ character for the enhancement of threonine productivity, and also the possibility of obtaining excellent threonine producers having both $\mathrm{HD}^{R}$ S and a DPS ${ }^{-}$or DPS ${ }^{\mathrm{L}} \mathrm{s}$ by deriving mutants resistant to higher concentrations of AHV.

Acknowledgment. The authors are indebted to Dr. I. Maeda of our laboratories for encouragement during this work.

\section{References}

1) I. Shiio and S. Nakamori, Agric. Biol. Chem., 34, 448 (1970).

2) I. Shiio, R. Miyajima and S. Nakamori, J. Biochem., 68, 859 (1970).

3) I. Shiio, A. Yokota, Y. Toride and S. Sugimoto, Agric. Biol. Chem., 53, 41 (1989).

4) I. Shiio, A. Yokota and K. Kawamura, Agric. Biol. Chem., 53, 2169 (1989).

5) I. Shiio, A. Yokota and S. Sugimoto, Agric. Biol. Chem., 51, 2485 (1987).

6) S. Nakamori and I. Shiio, Agric. Biol. Chem., 36, 1209 (1972).

7) R. Miyajima and I. Shiio, Agric. Biol. Chem., 34, $1275(1970)$. 